\title{
Intelligent numerical control power supply system design
}

\author{
Huaqun Zhan, Wenjie Wu, Yonghao Zhou
}

Jiangxi normal university of science and technology, Nanchang 330000, China

\begin{abstract}
Keywords: STC12C5616AD, Digitization, Voltage source.
\end{abstract}
\begin{abstract}
To control the output voltage by adjusting the size of the sliding rheostat, Aiming at the problem of its output precision and control, A digital adjustable power supply system based on single chip microcomputer is designed. System makes single chip microcomputer (STC5616AD) as the core controller, makes the button as the regulator, uses the crystal display technology, monitors the output voltage and current size at any time, The over current protection of the circuit is warned carried out by the over current alarm circuit, and provides users with visual information. The hardware complexity of the system is lower. The output voltage has good stability and high accuracy. It is convenient to regulate output voltage of constant voltage.
\end{abstract}

\section{Introduction}

Digital power supply changes the output voltage by means of controlling buttons to change the increase or decrease of the stepper motor. Many power supply changes the size of the regulator to control the output voltage. Both accuracy and stability are not well controlled. Without LCD display technology, system stability is very poor, without visual interface with the user, voltage regulation is not convenient. To solve the above problems, this paper designs a voltage source system which is easy to regulate output voltage and has constant voltage with the user interface. With the continuous development of electronic technology, more and more electronic products put forward higher requirements on accuracy and stability of power supply. Power supply develops towards the digital, modular, reliable, intelligent. This system is a voltage source with $0.07 \mathrm{~V}$ as the stepping value to ensure the output voltage value between 0 and $17.8 \mathrm{~V}$.

\section{Overall system design}

The system is composed of auxiliary power supply circuit, MCU control circuit, liquid crystal display circuit, PWM control output constant voltage circuit, current sampling circuit, output voltage sampling circuit and so on. STC5616AD microcontroller is the data processing module of the central processing unit. The system adjusts the output value by control button. The duty cycle of the PWM wave is controlled by a key press, which is formed DC by the two order low pass filter, the formed DC is used to control the working point of the composite pipe after the operational amplifier. The stable output of voltage is realized through the closed-loop negative feedback circuit. The system has a protective function for the output lock and output current, to prevent effectively the error caused by incorrect operation of the key. The overall structure of the system is shown in Figure 1.

\section{3. key hardware system design}

Key input circuit is shown in Figure 2.Use button set the circuit up to work: Press S1 (LOCK) to lock the keyboard and unlock the lock (every click, change once). Press S2 (OUT) can open and close the output voltage (every click, change once). In case of opening the output voltage, S4 (V+) and S3 (V-) keys are used to adjust the output voltage. The S6 (I+) and S5 (I-) buttons are used to adjust the output protection current, when the actual output current is bigger than the set protection current, circuit automatically shut off output voltage, and carry out sound and light alarm. 


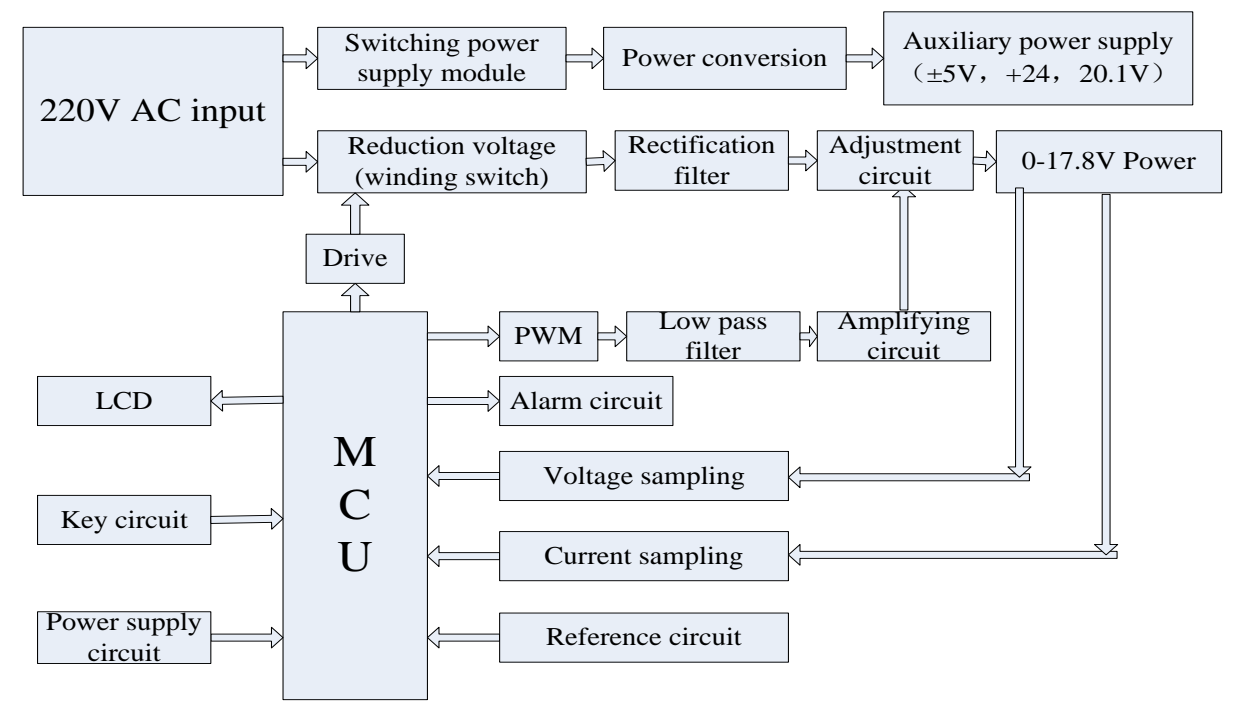

Figure 1 system structure diagram

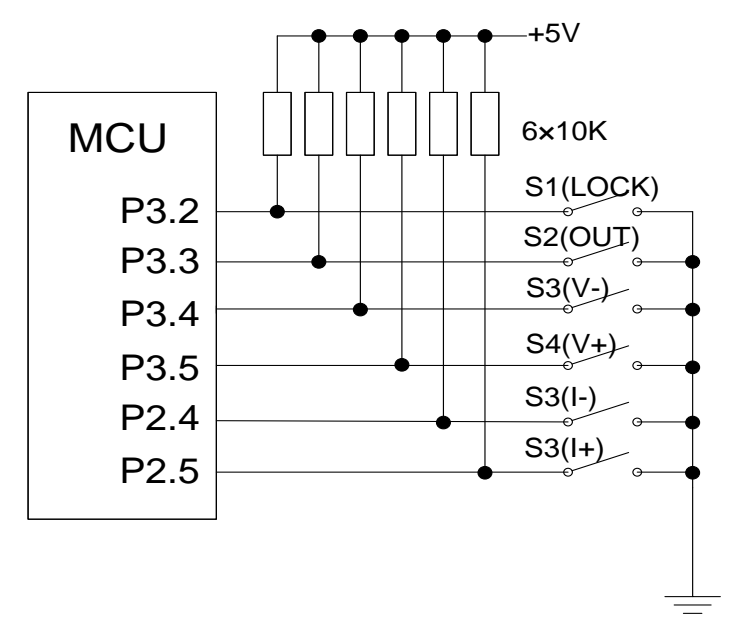

Figure 2 key input circuit

LCD

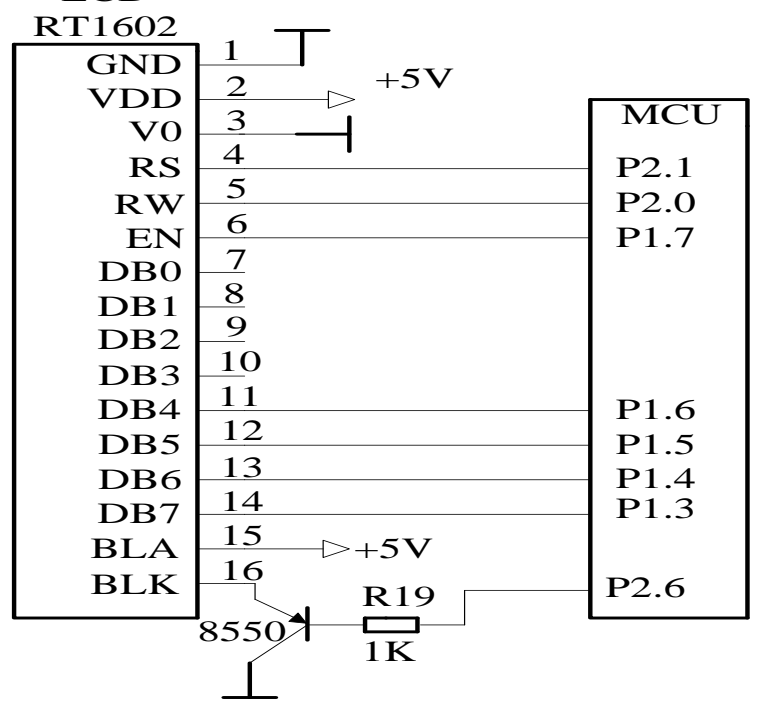

Figure 3 liquid crystal display circuit

LCD1602 has two control modes: 8 bit and 4 bit control mode. The 8 bit control mode is to use the D0-D7 data cable to send control command and data, the 4 bit control mode is to use the D4-D7 data cable to send control command and data. When using the 4 control mode, the data needs to be 
transmitted in two times, firstly sends 4 bit data, and then sends out the low 4 bit data, which can save 4 port line of the microcomputer. Due to the IO port of the design limited, we chooses the 4 control mode. Liquid crystal display circuit normally operates: LCD liquid crystal display can normally display the welcome interface, output voltage and state ,output current and protective current and so on.

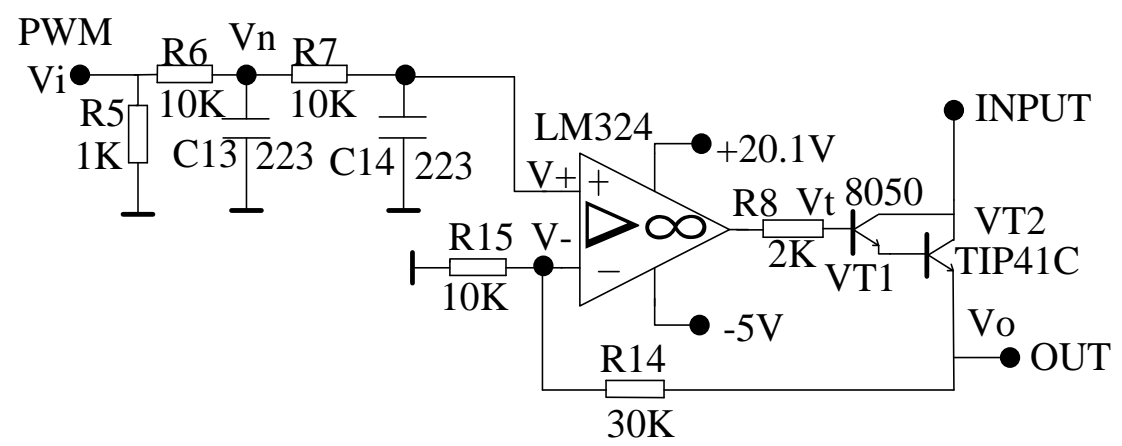

Figure 4 output steady voltage circuit

The circuit consists of two order low pass filter, amplifier and feedback network in Figure 4. In order to make the voltage drop faster in the high frequency range, we chooses the two order low-pass filter. Circuit chooses the noninverting input amplifier, who has very high impedance and low output impedance, can be widely used in pre amplifier.

Pass band gain

When the $\mathrm{f}=0$ or the frequency is very low, each capacitor is treated as an open circuit, the gain of the pass band is:

$A_{V}=1+R_{1} \& R_{1} 5$

Transfer function

By C13=C14=C, R6=R7=R, we can get

$$
\begin{aligned}
& V_{N}(s)=\frac{\frac{1}{s C} / /\left(R+\frac{1}{s C}\right)}{R+\left[\frac{1}{s C} / /\left(R+\frac{1}{s C}\right)\right]} V_{i}(s) \\
& V_{(+)}(s)=V_{N}(s) \frac{1}{1+s C R} \\
& V_{o}(s)=A_{V P} V_{(+)}(s)
\end{aligned}
$$

The transfer function of simultaneous can be get by (2), (3), (4)

$A_{v}(s)=\frac{V_{o}(s)}{V_{i}(s)}=\frac{A_{v p}}{1+3 s C R+(s C R)^{2}}$

(3) Cut off frequency of the pass band

Change s into $j \omega$, order $\omega_{0}=2 \pi \mathrm{f}_{0}=1 / \mathrm{RC}$, we can get

$$
\dot{A}_{\mathrm{v}}=\frac{A_{v p}}{1-\left(\frac{f}{f_{0}}\right)^{2}+j 3 \frac{f}{f_{0}}}
$$

When $f=f_{p}$ S, the cutoff frequency can be received by the model of the denominator $\left|1-\left(\frac{f}{f_{0}}\right)^{2}+j 3 \frac{f}{f_{0}}\right|=\sqrt{2}$

$$
f_{p}=\sqrt{\frac{\sqrt{53}-7}{2}} f_{0}=0.37 f_{0}=\frac{0.37}{2 \pi R C}
$$

Beyond $f_{0}$, amplitude frequency descends at the rate of $-40 \mathrm{~dB} / \mathrm{dec}$, which is faster than the first order.

The gain of the amplifier circuit consisted by VT2 and VT1 is:

$\beta=\beta_{V \text { 11 }} \beta_{\text {V य }}$ 
PWM square wave with a certain duty cycle of $\alpha$ can be get through adjusting press key. After two - stage low pass filter the circuit outputs proportional DC voltage $V_{+}=\left(V_{H}-V_{L}\right) \times \alpha$. According to the deduced form of the ideal operational amplifier.

$$
V-=V+\quad \therefore \mathrm{V} \mathrm{o}=A_{v p} \times V-
$$

The composite pipe breakover at this time, output voltage changes are as follows

$$
V_{o} \uparrow \rightarrow \mathrm{V}-\uparrow \rightarrow \mathrm{V}->\mathrm{V}_{+} \rightarrow V_{t}=-V_{C C}<0 \rightarrow V_{o} \downarrow \rightarrow \mathrm{V}-\downarrow \rightarrow \mathrm{V}-<\mathrm{V}_{+} \rightarrow V_{t}=V_{o}+1.4 V>0 \rightarrow V_{o} \uparrow
$$

Through above, the output voltage is stable at a value by adjusting the button. Another advantage of this design is whether external load pulls low the voltage or the external interference changes the output voltage value, the circuit will stable output voltage at the preset value.

\section{4. software system design}

The software design of the system includes: initialization of the single chip microcomputer, key scan program design, program design of key scan, program design of liquid crystal display, algorithm implementation of 4 liquid crystal control mode, program design of analog to digital conversion. The flow chart of the system is shown in Figure 6.

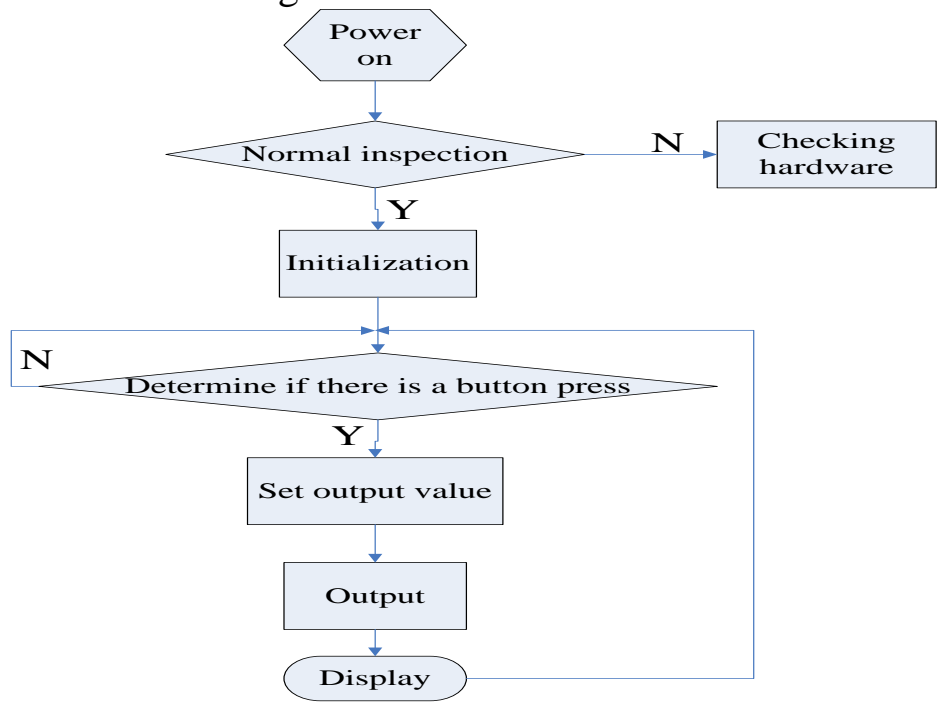

Figure 5 software flow chart

When the power is turned on, the MCU is reset and the register is cleared. See whether the hardware module normally works after $50 \mathrm{~ms}$ of powering on. Then single chip microcomputer initializes LCD and ADC module, and selects the mode of ADC works. The welcome interface can be seen on the LCD display, which indicates LCD setting the right and working as well. Single chip microcomputer scans the keys to find which keys are pressed and does the appropriate operation. The set output voltage are sent to ADC module of the microcontroller after through the voltage acquisition module. The data is displayed in the liquid crystal display after transformed into corresponding digital data. At this time, the program cycle checks wehther there is a button has been pressed down, if $\mathrm{S} 1$ is pressed, the output voltage is to be locked so as not to affect the output voltage due to the jitter of the other keys, if S2 is pressed, the voltage output can be controlled.

The following algorithm is used for the 4 bit control of LCD

unsigned char change_byte(unsigned char byte)

\{

Unsigned char byte1=0xff, i, b;

For $(\mathrm{i}=0 ; \mathrm{i}<8 ; \mathrm{i}++)$

\{

Byte $<<=1$; //remove the highest level of data

If $(\mathrm{CY}==1) \mathrm{b}=0 \mathrm{xff}$; // determine whether the carry is 1

Else if $(C Y==0) b=0 x 7 f$; //determine whether the carry is 0 
byte1=byte1\&b;

if (i<7)byte1=_cror_(byte1,1);//Correspondingly rotate right obtained data

\}

Return byte1;

\}

From Figure 3 we can see that the high four post of LCD is connected with I/O P1.3 to the P1.6 of the chip, and the relative position is just the opposite. Data of high and low level of data can be exchanged.

\section{5. system testing}

In this system, the modules of the circuit are tested to make sure each works properly, then combine test to detect whether they meet the design requirement. The test items include input and output voltage range, step value of the key adjustment, ripple measurement and output current value.

\section{1 input and output voltage range and output current measurement}

Use AC voltage profile of the multimete to measure input voltage, use dc gear to measure output voltage, use DC current profile to measure output current flowed through load. The input voltage is $220 \mathrm{~V}$, output voltage changes between $0-17.8 \mathrm{~V}$, output rated operating current is $2.0 \mathrm{~A}$, those meet the design requirements of the system.

\section{2 output voltage step value and ripple measurement}

Measure the output voltage by using the DC file and AC file of the oscilloscope.

The minimum step value is $0.07 \mathrm{~V}$ through regulating the buttons of the $\mathrm{V}+$ and $\mathrm{V}$-, the size of the ripple is $26 \mathrm{mV}$ (Less than $70 \mathrm{mV}$ ), all above meet the requirements of the design.

\section{Summary}

This paper introduces a design scheme of digital power supply based on single chip microcomputer, focus on the overall design scheme and the design of the hardware circuit. The numerical control voltage source adopts STC12C5616AD single chip microcomputer as the control unit, this controller integrates the ADC converter module and the comparator module, which greatly reduces the complexity and the design cost of the hardware structure of the system. The output voltage, locks button, controls output and over current protection can be set by the key. After setting the value of the pre input by the key, single chip outputs setted the duty cycle of the PWM wave, then formates a voltage proportional to the duty ratio of PWM through the low pass filter, then gains output voltage equalling four times the voltage value of the same phase input terminal through the amplifier and composite tube amplifier. The digital data after voltage acquisition is sent to the he ADC module of the MCU, which is convenient to output voltage value by Software fine tuning. Through practical use, this design has the advantages of setting conveniently the output voltage, good stability and high accuracy. This system is easy to upgrade and promote and simple hardware design.

\section{Reference}

[1]Shibai Tong,Chenying Hua. Basic analog electronics[M], 2009.

[2]Xiaojun Jing,Jianyong Li,Fengjie Ji. Precision numerical control power supply design[J]. Power the world, 2011(1).

[3]Yigang Zhang. Single chip microcomputer principle and application[M]. Higher education press, 2010.

[4]Guixian Zhu. Design of digital power supply based on single chip microcomputer[J]. Automation and instrumentation, 2011(6):50-53.

[5]ShiYan. Digital electronic technology foundation[M]. Higher education press,2009. 